\title{
EPISTURMIAN MORPHISMS AND A GALOIS THEOREM ON CONTINUED FRACTIONS
}

\author{
JACQUES JUSTIN ${ }^{1,2}$
}

\begin{abstract}
We associate with a word $w$ on a finite alphabet $A$ an episturmian (or Arnoux-Rauzy) morphism and a palindrome. We study their relations with the similar ones for the reversal of $w$. Then when $|A|=2$ we deduce, using the Sturmian words that are the fixed points of the two morphisms, a proof of a Galois theorem on purely periodic continued fractions whose periods are the reversal of each other.
\end{abstract}

Mathematics Subject Classification. 11A55, 68R15.

\section{INTRODUCTION}

Sturmian words on a two-letter alphabet have been actively studied, at least since the fundamental paper of Morse and Hedlund [15]. For a survey, see [13]. These words have a deep relation with simple continued fractions. Among the generalizations of Sturmian words to any finite alphabet, the one known as ArnouxRauzy sequences $[2,16-18]$ or, with even slightly more generality, as episturmian words $[3,8,11,12]$ leads to many properties extending those of Sturmian words. In particular the continued fractions point of view has a transposition for episturmian words [20]. In this paper the famous Lagrange theorem relating periodic continued fractions to quadratic numbers was extended to a "multidimensional" continued fraction algorithm (see also [19]). However this one has not the same efficiency as the classical one for approximating irrationals by rationals. Evariste Galois

\footnotetext{
Keywords and phrases. Episturmian morphism, Arnoux-Rauzy morphism, palindrome, continued fraction, Sturmian word.

${ }^{1}$ Present address: 19 rue de Bagneux, 92330 Sceaux, France.

2 LIAFA, ERS 586, Université Paris VII, case 7014, 2 place Jussieu, 75251 Paris Cedex 5, France; justin@liafa.jussieu.fr
} 
found, when a student at Lycée Louis le $\mathrm{Grand}^{1}$, a theorem relating the values of two purely periodic (simple) continued fractions whose periods are reversal of each other [9]. In the same paper Galois also gives a characterization of quadratic numbers with a purely periodic continued fraction expansion. Both results are used in [1] for characterizing the so-called Sturm numbers (see also [13], Th. 2.3.26).

Here we study relations involving some (finite) episturmian words and episturmian morphisms. More precisely we associate with a (finite) word $w$ a palindrome $\operatorname{Pal}(w)$ and a morphism $\mu_{w}$. Such palindromes (which are the bispecial Arnoux-Rauzy words of [14]) play an important role in the theory of (infinite) episturmian words [8], in particular in the Sturmian case where they are called central words [13].

In Section 2 we establish some relations between $\operatorname{Pal}(w), \mu_{w}, \operatorname{Pal}(\tilde{w}), \mu_{\tilde{w}}$, where $\tilde{w}$ is the reversal of $w$. These relations are explained by the fact that the incidence matrices of $\mu_{w}$ and $\mu_{\tilde{w}}$ are similar.

In Section 3, applying this to a 2-letter alphabet, we prove the above-mentioned Galois theorem by considering the standard episturmian words which are the fixed points of $\mu_{w}$ and $\mu_{\tilde{w}}$.

It seems to us that this confirms the interest of the multidimensional continued fraction algorithm in the above sense. However it remains much work to do on the subject, in particular in relation with the generalized intercept introduced in [11] and the generalized Ostrowski numeration systems $[4,12]$.

\section{Preliminaries}

The alphabet $A,|A| \geq 2$, is finite and will be kept throughout the paper, $\varepsilon$ is the empty word, $A^{*}$ is the set of (finite) words and $A^{\omega}$ the set of (right) infinite words on $A$.

The length of $v=x_{1} \cdots x_{n}, x_{i} \in A$, is $|v|=n$ and the number of occurences of letter $x$ in $v$ is $|v|_{x}$. If $|v|_{x}=0$, then $v$ is $x$-free. The reversal of $v$ is $\tilde{v}=x_{n} \cdots x_{1}$ and $v$ is a palindrome if $\tilde{v}=v$.

If $v \in A^{*}$ the (Parikh) vector of $v$ is the $|A| \times 1$ vector of the $|v|_{x}, x \in A$, and if $\varphi$ is an (endo)morphism of $A^{*}$ its incidence matrix is the $|A| \times|A|$ matrix $M=\left(m_{x y}\right)$ whose columns are the vectors of the $|\varphi(y)|, y \in A$, i.e., $m_{x y}=|\varphi(y)|_{x}$.

For $u \in A^{*}$ its (right) palindromic closure is the shortest palindrome $u^{(+)}$having $u$ as a prefix. We have $u^{(+)}=u v^{-1} u$ with $v$ the longest palindromic suffix of $u$ (when $h=f g$ we sometimes write $f=h g^{-1}$ and $g=f^{-1} h$ ).

Now we say some words about (infinite) episturmian words, limiting ourselves to what is really needed here. Let $\Delta \in A^{\omega}, \Delta=x_{1} x_{2} \cdots x_{i} \cdots, x_{i} \in A$. The infinite word having the sequence of prefixes $u_{1}=\varepsilon, u_{2}=x_{1}, \ldots, u_{i+1}=\left(u_{i} x_{i}\right)^{(+)}, \ldots$ is the standard episturmian word directed by $\Delta$ (also called characteristic Arnoux-Rauzy

\footnotetext{
1 The author also studied at Lycée Louis le Grand but without discovering any theorem.
} 
sequence if $\Delta$ contains infinitely many occurrences of each of its letters). In particular for $|A|=2$ Arnoux-Rauzy sequences are the Sturmian words, while episturmian words also include the "periodic Sturmian words", i.e., the infinite words given by cutting sequences with rational slope.

Now let $w=x_{1} \cdots x_{n}, x_{i} \in A$, then using the successive letters of $w$ construct as previously the sequence $u_{1}=\varepsilon, \ldots, u_{n+1}=\left(u_{n} x_{n}\right)^{(+)}$of all palindromic prefixes of $u_{n+1}$. We write $u_{n+1}=\operatorname{Pal}(w)$ and say that $w$ directs Pal $(w)$. Let $x \in A$. If $w$ is $x$-free then $\operatorname{Pal}(w x)=\operatorname{Pal}(w) x \operatorname{Pal}(w)$. If $x$ occurs in $w$ write $w=w_{1} x w_{2}$ with $w_{2} x$-free. Then the longest palindromic prefix of $\operatorname{Pal}(w)$ followed by $x$ in $\operatorname{Pal}(w)$ is $\operatorname{Pal}\left(w_{1}\right)$ whence easily

$$
\operatorname{Pal}(w x)=\operatorname{Pal}(w) \operatorname{Pal}\left(w_{1}\right)^{-1} \operatorname{Pal}(w) .
$$

Consider for any letter $a$ the morphism $\psi_{a}$ such that $\psi_{a}(a)=a$ and $\psi_{a}(b)=a b$ for any letter $b \neq a$. The $\psi_{a}$ generate by composition the monoid of pure standard episturmian morphisms [11], Section 2.1.

For any $w=x_{1} \cdots x_{n}$ as previously we write $\mu_{w}=\psi_{x_{1}} \circ \psi_{x_{2}} \cdots \circ \psi_{x_{n}}$. In particular $\mu_{\varepsilon}$ is the identity and, for $x \in A, \mu_{x}=\psi_{x}$. We also denote by $M_{w}$ the incidence matrix of $\mu_{w}$.

The relations between $w, \mu_{w}, M_{w}$ are one-to-one and indeed are isomorphisms between $A^{*}$, the monoid of pure standard episturmian morphisms and the monoid of the $M_{w}$.

For continued fractions see [5] for instance.

\section{WORDS AND MATRICES RELATIONS}

The first lemma recalls and proves for sake of completeness some relations appearing with different notations in $[8,11]$. This allows to prove the curious relation $|\operatorname{Pal}(w)|=|\operatorname{Pal}(\tilde{w})|$ and some other ones. Then we give an interpretation in terms of the matrices $M_{w}$ and $M_{\tilde{w}}$.

\section{Lemma 2.1.}

1) For any palindrome $p$ and letter $x, \mu_{x}(p) x$ is a palindrome.

2) For any $w \in A^{*}, x \in A$ we have

$$
\operatorname{Pal}(x w)=\mu_{x}(\operatorname{Pal}(w)) x .
$$

3) For any $v, w \in A^{*}$,

$$
\operatorname{Pal}(v w)=\mu_{v}(\operatorname{Pal}(w)) \operatorname{Pal}(v) .
$$

Proof.

Part 1) follows from the easy fact that, for $u \in A^{*}, x \in A, \mu_{x}(\tilde{u}) x=\widetilde{x \mu_{x}(u)}$.

Part 2) is proved by induction on $|w|$. If $|w|=0$ the assertion is trivial. Otherwise let $w=v y, y \in A$. If $y$ occurs in $v$ write $v=v_{1} y v_{2}$ with $v_{2} y$-free. Then by 
equation $(1) \operatorname{Pal}(w)=\operatorname{Pal}(v) \operatorname{Pal}\left(v_{1}\right)^{-1} \operatorname{Pal}(v)$ whence

$$
\mu_{x}(\operatorname{Pal}(w)) x=\mu_{x}(\operatorname{Pal}(v)) x x^{-1} \mu_{x}\left(\operatorname{Pal}\left(v_{1}\right)\right)^{-1} \mu_{x}(\operatorname{Pal}(v)) x .
$$

As $|v|<|w|$ and $\left|v_{1}\right|<|w|$ we get by induction hypothesis

$$
\mu_{x}(\operatorname{Pal}(w)) x=\operatorname{Pal}(x v) \operatorname{Pal}\left(x v_{1}\right)^{-1} \operatorname{Pal}(x v) .
$$

As $x v=x v_{1} y v_{2}$ and $\left|v_{2}\right|_{y}=0$, equation (1) shows that the right member is $\operatorname{Pal}(x w)$.

If on the contrary $|v|_{y}=0$ then $\operatorname{Pal}(w)=\operatorname{Pal}(v) y \operatorname{Pal}(v)$ whence

$$
\mu_{x}(\operatorname{Pal}(w)) x=\mu_{x}(\operatorname{Pal}(v)) x x^{-1} \mu_{x}(y) \mu_{x}(\operatorname{Pal}(v)) x=\operatorname{Pal}(x v) x^{-1} \mu_{x}(y) \operatorname{Pal}(x v) .
$$

Thus if $y \neq x$ then $|w|_{y}=0$, whence $\mu_{x}(\operatorname{Pal}(w)) x=\operatorname{Pal}(x v) y \operatorname{Pal}(x v)=$ $\operatorname{Pal}(x v y)=\operatorname{Pal}(x w)$. If $y=x$ then similarly $\mu_{x}(\operatorname{Pal}(w)) x=\operatorname{Pal}(x v) \operatorname{Pal}(x v)=$ $\operatorname{Pal}(x v x)=\operatorname{Pal}(x w)$.

For 3) the proof is by induction on $|v|$. The assertion is trivial for $|v|=0$. Otherwise let $v=y v_{1}$. Using part 2) of the lemma and the induction hypothesis we successively get

$$
\begin{aligned}
\operatorname{Pal}(v w)=\operatorname{Pal}\left(y v_{1} w\right) & =\mu_{y}\left(\operatorname{Pal}\left(v_{1} w\right)\right) y=\mu_{y}\left(\mu_{v_{1}}(\operatorname{Pal}(w)) \operatorname{Pal}\left(v_{1}\right)\right) y \\
& =\mu_{y v_{1}}(\operatorname{Pal}(w)) \mu_{y}\left(\operatorname{Pal}\left(v_{1}\right)\right) y=\mu_{v}(\operatorname{Pal}(w)) \operatorname{Pal}(v) .
\end{aligned}
$$

Lemma 2.2. For $w \in A^{*}$ if $x \in A$ occurs in $w$ write $w=w_{1} x w_{2}$ with $w_{1} x$-free. Then

$$
|\operatorname{Pal}(w)|_{x}=\left|\operatorname{Pal}\left(w_{2}\right)\right|+1 .
$$

Proof. By Lemma 2.1 $\operatorname{Pal}(w)=\mu_{w_{1}}\left(\operatorname{Pal}\left(x w_{2}\right)\right) \operatorname{Pal}\left(w_{1}\right)$ whence as $w_{1}$ is $x$-free

$$
\begin{aligned}
|\operatorname{Pal}(w)|_{x} & =\left|\mu_{w_{1}}\left(\operatorname{Pal}\left(x w_{2}\right)\right)\right|_{x}=\left|\operatorname{Pal}\left(x w_{2}\right)\right|_{x} \\
& =\left|\mu_{x}\left(\operatorname{Pal}\left(w_{2}\right)\right) x\right|_{x}=\left|\operatorname{Pal}\left(w_{2}\right)\right|+1 .
\end{aligned}
$$

We deduce a rather curious relation between the palindromes directed by $w$ and $\tilde{w}$.

Theorem 2.3. For any $w \in A^{*}, \operatorname{Pal}(w)$ and $\operatorname{Pal}(\tilde{w})$ have the same length.

Proof. The proof is by induction on $|w|$. This is trivial for $|w|=0$. Otherwise set $w=v x$. If $v$ is $x$-free then $\operatorname{Pal}(w)=\operatorname{Pal}(v) x \operatorname{Pal}(v)$ whence $|\operatorname{Pal}(w)|=$ $2|\operatorname{Pal}(v)|+1$. Also, by equation $(2), \operatorname{Pal}(\tilde{w})=\operatorname{Pal}(x \tilde{v})=\mu_{x}(\operatorname{Pal}(\tilde{v})) x$. As $\operatorname{Pal}(\tilde{v})$ is $x$-free, $\left|\mu_{x}(\operatorname{Pal}(\tilde{v}))\right|=2|\operatorname{Pal}(\tilde{v})|$. Thus using the induction hypothesis $|\operatorname{Pal}(\tilde{w})|=2|\operatorname{Pal}(\tilde{v})|+1=2|\operatorname{Pal}(v)|+1=|\operatorname{Pal}(w)|$.

Otherwise $x$ occurs in $v$. Write $v=v_{1} x v_{2}$ with $v_{2} x$-free. Then by equation (1) $\operatorname{Pal}(w)=\operatorname{Pal}(v x)=\operatorname{Pal}(v) \operatorname{Pal}\left(v_{1}\right)^{-1} \operatorname{Pal}(v)$ whence $|\operatorname{Pal}(w)|=2|\operatorname{Pal}(v)|-$ $\left|\operatorname{Pal}\left(v_{1}\right)\right|$. 
Also $\operatorname{Pal}(\tilde{w})=\operatorname{Pal}(x \tilde{v})=\mu_{x}(\operatorname{Pal}(\tilde{v})) x$ whence $|\operatorname{Pal}(\tilde{w})|=2|\operatorname{Pal}(\tilde{v})|-$ $|\operatorname{Pal}(\tilde{v})|_{x}+1$. But Lemma 2.2 applied to $\tilde{v}=\tilde{v}_{2} x \tilde{v}_{1}$ gives $|\operatorname{Pal}(\tilde{v})|_{x}=\left|\operatorname{Pal}\left(\tilde{v}_{1}\right)\right|+1$. Thus using induction hypothesis,

$$
|\operatorname{Pal}(\tilde{w})|=2|\operatorname{Pal}(\tilde{v})|-\left|\operatorname{Pal}\left(\tilde{v}_{1}\right)\right|=2|\operatorname{Pal}(v)|-\left|\operatorname{Pal}\left(v_{1}\right)\right|=|\operatorname{Pal}(w)| .
$$

Lemma 2.4. Let $w \in A^{*}, y \in A$.

1) If $w$ is $y$-free then $\mu_{w}(y)=\operatorname{Pal}(w) y$. Otherwise write $w=v_{1} y v_{2}, v_{2} y$-free, then $\mu_{w}(y)=\operatorname{Pal}(w) \operatorname{Pal}\left(v_{1}\right)^{-1}$.

2) With $x \in A$, if $w$ is $x$-free or $y$-free then $\left|\mu_{w}(y)\right|_{x}=|\operatorname{Pal}(w)|_{x}+|y|_{x}$. Otherwise write $w=v_{1} y v_{2}=w_{1} x w_{2}$ with $\left|v_{2}\right|_{y}=\left|w_{1}\right|_{x}=0$. Then

$$
\left|\mu_{w}(y)\right|_{x}=|\operatorname{Pal}(w)|_{x}-\left|\operatorname{Pal}\left(v_{1}\right)\right|_{x}=|\operatorname{Pal}(w)|_{x}-\left|\operatorname{Pal}\left(\tilde{w}_{2}\right)\right|_{y} .
$$

Proof. For 1), by equation (3), $\operatorname{Pal}(w y)=\mu_{w}(y) \operatorname{Pal}(w)$. If $w$ is $y$-free then $\operatorname{Pal}(w y)=\operatorname{Pal}(w) y \operatorname{Pal}(w)$ whence $\mu_{w}(y)=\operatorname{Pal}(w) y$. Otherwise, with $w=v_{1} y v_{2}$, $v_{2} y$-free, $\operatorname{Pal}(w y)=\operatorname{Pal}(w) \operatorname{Pal}\left(v_{1}\right)^{-1} \operatorname{Pal}(w)$ whence $\mu_{w}(y)$ as claimed.

For 2), if $w$ is $y$-free then $\left|\mu_{w}(y)\right|_{x}=|\operatorname{Pal}(w) y|_{x}=|\operatorname{Pal}(w)|_{x}+|y|_{x}$. If $w$ is $x$-free and $x \neq y$ then $\left|\mu_{w}(y)\right|_{x}=0=|\operatorname{Pal}(w)|_{x}+|y|_{x}$.

Otherwise, write $w=v_{1} y v_{2}=w_{1} x w_{2}$ with $\left|v_{2}\right|_{y}=\left|w_{1}\right|_{x}=0$. Then $\mu_{w}(y)=$ $\operatorname{Pal}(w) \operatorname{Pal}\left(v_{1}\right)^{-1}$ whence $\left|\mu_{w}(y)\right|_{x}=|\operatorname{Pal}(w)|_{x}-\left|\operatorname{Pal}\left(v_{1}\right)\right|_{x}$.

It remains to show that $\left|\operatorname{Pal}\left(v_{1}\right)\right|_{x}=\left|\operatorname{Pal}\left(\tilde{w}_{2}\right)\right|_{y}$. If $\left|v_{1}\right| \leq\left|w_{1}\right|$ then $v_{1}$ is $x$-free, $w_{2}$ is $y$-free, hence $\left|\operatorname{Pal}\left(v_{1}\right)\right|_{x}=\left|\operatorname{Pal}\left(\tilde{w}_{2}\right)\right|_{y}=0$. Otherwise, $\left|v_{1}\right|>\left|w_{1}\right|$. Write $v_{1}=w_{1} x u, u \in A^{*}$. Then using Lemma 2.2, as $v_{2}$ is $y$-free and $w_{1}$ is $x$-free, $\left|\operatorname{Pal}\left(v_{1}\right)\right|_{x}=|\operatorname{Pal}(u)|+1$ and similarly $\left|\operatorname{Pal}\left(\tilde{w}_{2}\right)\right|_{y}=|\operatorname{Pal}(\tilde{u})|+1=|\operatorname{Pal}(u)|+1$.

Corollary 2.5. The traces of $M_{w}$ and $M_{\tilde{w}}$ are equal.

Proof. For $x \in A$, if $|w|_{x}=0$ then $\left|\mu_{w}(x)\right|_{x}=\left|\mu_{\tilde{w}}(x)\right|_{x}=1$. Otherwise let $w=v_{1} x v_{2}=w_{1} x w_{2}$ with $\left|v_{2}\right|_{x}=\left|w_{1}\right|_{x}=0$. Then by Lemma $2.4\left|\mu_{w}(x)\right|_{x}=$ $|\operatorname{Pal}(w)|_{x}-\left|\operatorname{Pal}\left(v_{1}\right)\right|_{x}=|\operatorname{Pal}(w)|_{x}-\left|\operatorname{Pal}\left(\tilde{w}_{2}\right)\right|_{x}$. Similarly $\left|\mu_{\tilde{w}}(x)\right|_{x}=|\operatorname{Pal}(\tilde{w})|_{x}-$

$\left|\operatorname{Pal}\left(\tilde{w}_{2}\right)\right|_{x}$. Thus in both cases $\left|\mu_{w}(x)\right|_{x}-\left|\mu_{\tilde{w}}(x)\right|_{x}=|\operatorname{Pal}(w)|_{x}-|\operatorname{Pal}(\tilde{w})|_{x}$.

Summing over $x \in A$ we get $\operatorname{tr}\left(M_{w}\right)-\operatorname{tr}\left(M_{\tilde{w}}\right)=|\operatorname{Pal}(w)|-|\operatorname{Pal}(\tilde{w})|=0$.

It is possible from this to show that $M_{w}$ and $M_{\tilde{w}}$ have the same eigenvalues. Indeed $M_{w^{k}}$ and $M_{\tilde{w}^{k}}$ have the same traces for any integer $k$. As the trace is the sum of the eigenvalues and as the eigenvalues of $M_{w^{k}}$ and $M_{\tilde{w}^{k}}$ are the $k$-th powers of those of $M_{w}$ and $M_{\tilde{w}}$ we get that the Newton sums of the eigenvalues of $M_{w}$ and $M_{\tilde{w}}$ are the same and that these matrices have the same characteristic polynomial.

Theorem 2.6. For $x \in A$ set $L_{w}(x)=\sum_{y \in A}\left|\mu_{w}(y)\right|_{x}$. Then

$$
L_{w}(x)=(|A|-1)|\operatorname{Pal}(w)|_{x}+1 .
$$

Proof. If $|w|_{x}=0$ then $L_{w}(x)=\left|\mu_{w}(x)\right|_{x}=1=(|A|-1)|\operatorname{Pal}(w)|_{x}+1$. Otherwise let $w=w_{1} x w_{2}$ with $w_{1} x$-free. Then by Lemma 2.4, 2) $\left|\mu_{w}(y)\right|_{x}=$ $|\operatorname{Pal}(w)|_{x}-\left|\operatorname{Pal}\left(\tilde{w}_{2}\right)\right|_{y}$. Summing over $y$ we get $L_{w}(x)=|A||\operatorname{Pal}(w)|_{x}-\left|\operatorname{Pal}\left(\tilde{w}_{2}\right)\right|$. 
As by Lemma $2.2\left|\operatorname{Pal}\left(\tilde{w}_{2}\right)\right|=\left|\operatorname{Pal}\left(w_{2}\right)\right|=|\operatorname{Pal}(w)|_{x}-1$ we get $L_{w}(x)=$ $(|A|-1)|\operatorname{Pal}(w)|_{x}+1$.

Corollary 2.7.

$$
\sum_{y \in A}\left|\mu_{w}(y)\right|=(|A|-1)|\operatorname{Pal}(w)|+|A| .
$$

Proof. By summation of equation (5) over $x$.

Remark 2.1. The $\left|\mu_{w}(y)\right|$ are periods of $\operatorname{Pal}(w)$ and indeed formula (6) is the same as the one given in [7] for $|A|=3$ and [10] for $|A| \geq 2$. More precisely the ordered set of the $\left|\mu_{w}(y)\right|$ is a "good" $|A|$-uple in the sense of these papers with its GCD equal to 1 and $\operatorname{Pal}(w)$ is a word of maximal length having these periods $\left|\mu_{w}(y)\right|$ and not having period 1 (this is a particular case of the Fine and Wilf theorem for $|A|$ periods).

Proposition 2.8. For any $x, y \in A$

$$
L_{w}(x)-L_{\tilde{w}}(y)=(|A|-1)\left(\left|\mu_{w}(y)\right|_{x}-\left|\mu_{\tilde{w}}(x)\right|_{y}\right)
$$

Proof. By Theorem 2.6, $L_{w}(x)-L_{\tilde{w}}(y)=(|A|-1)\left(|\operatorname{Pal}(w)|_{x}-|\operatorname{Pal}(\tilde{w})|_{y}\right)$. If $|w|_{x}=0$ or $|w|_{y}=0$ then by Lemma 2.4, 2) $\left|\mu_{w}(y)\right|_{x}=|\operatorname{Pal}(w)|_{x}+|y|_{x}$ and $\left|\mu_{\tilde{w}}\right|_{y}=|\operatorname{Pal}(\tilde{w})|_{y}+|x|_{y}$ whence the result. Otherwise let $w=w_{1} x w_{2}$ with $\left|w_{1}\right|_{x}=0$. Then by Lemma 2.4,2) $\left|\mu_{w}(y)\right|_{x}=|\operatorname{Pal}(w)|_{x}-\left|\operatorname{Pal}\left(\tilde{w}_{2}\right)\right|_{y}$ and $\left|\mu_{\tilde{w}}(x)\right|_{y}=|\operatorname{Pal}(\tilde{w})|_{y}-\left|\operatorname{Pal}\left(\tilde{w}_{2}\right)\right|_{y}$ whence the result.

Example 2.1. With $A=\{1,2,3\}, w=123^{2} 2^{3}, \tilde{w}=2^{3} 3^{2} 21$,

$$
\begin{gathered}
\operatorname{Pal}(w)=12131213121213121312121312131212131213121 \\
\operatorname{Pal}(\tilde{w})=22232223222232223222122232223222232223222,
\end{gathered}
$$

both of length 41 , and

$$
M_{w}=\left(\begin{array}{ccc}
21 & 5 & 17 \\
12 & 3 & 10 \\
8 & 2 & 7
\end{array}\right) \quad M_{\tilde{w}}=\left(\begin{array}{ccc}
1 & 1 & 1 \\
16 & 23 & 26 \\
4 & 6 & 7
\end{array}\right) .
$$

It is possible to write Proposition 2.8 as a matricial relation, but we will see now a more direct way.

Theorem 2.9. Matrices $M_{w}$ and $M_{\tilde{w}}$ are similar and are related by

$$
H M_{w}=\left(H M_{\tilde{w}}\right)^{\mathrm{T}}
$$

with $H=\left(h_{x y}\right)$ given by $h_{x x}=0, h_{x y}=1, x, y \in A, x \neq y$.

Proof. For any letter $x$ it is immediate to verify that $H M_{x}=\left(H M_{x}\right)^{\mathrm{T}}$ whence $(7)$ by product. 
Remark 2.2. Let $K=\left(k_{x y}\right)$ such that, $k_{x x}=2-|A|, k_{x y}=1, x, y \in A, x \neq y$, then $H K=K H=(|A|-1) \mathrm{I}$ whence

$$
M_{\tilde{w}}^{\mathrm{T}}=(|A|-1)^{-1} H M_{w} K .
$$

It is possible to deduce from Theorem 2.9 some relations given above and even some other ones due to the particular form of $H$, for example $\operatorname{det}\left(M_{w}-M_{\tilde{w}}^{T}\right)=0$ for $|A|>2$ and $\operatorname{det}\left(M_{w}-M_{\tilde{w}}\right)=0$ for odd $|A|$.

Corollary 2.10. The word $w$ is a palindrome if and only if the matrix $H M_{w}$ is symmetrical.

Proof. By Theorem $2.9 H M_{w}$ is symmetrical if and only if $M_{w}=M_{\tilde{w}}$. Thus, in view of the bijection between $w$ and $M_{w}$ the proof is over.

Remark 2.3. When $A$ is a 2-letter alphabet, $\{1,2\}$, [6] gives a number theoretical condition on the $\left|\mu_{w}(y)\right|$ equivalent to $w$ is a palindrome, namely $\left|\mu_{w}(1)\right|^{2} \equiv 1$ $\bmod \left(\left|\mu_{w}(1)\right|+\left|\mu_{w}(2)\right|\right)$. It could be asked whether this condition can be extended to any finite alphabets.

\section{The CASE $|A|=2$ AND A Galois THEOREM}

From now on $A=\{1,2\}$. Set $M_{w}=\left(m_{i j}\right), M_{\tilde{w}}=\left(m_{i j}^{\prime}\right), 1 \leq i, j \leq 2$. For short we also set $p_{1}=m_{11}+m_{21}, p_{2}=m_{12}+m_{22}$ and similarly $p_{1}^{\prime}=m_{11}^{\prime}+m_{21}^{\prime}$, $p_{2}^{\prime}=m_{12}^{\prime}+m_{22}^{\prime}$.

The matrix $H$ of Theorem 2.9 becomes the permutation matrix $\left(\begin{array}{ll}0 & 1 \\ 1 & 0\end{array}\right)$, thus by this theorem $M_{\tilde{w}}=\left(\begin{array}{ll}m_{22} & m_{12} \\ m_{21} & m_{11}\end{array}\right)$.

Consider an infinite simple continued fraction, $\left[e_{1}, e_{2}, \ldots\right], e_{1} \geq 0, e_{2}, e_{3}, \ldots>0$ and the standard Sturmian infinite word $s$ directed by $\Delta=1^{e_{1}} 2^{e_{2}} 1^{e_{3}} \ldots$. Let $\alpha_{1}, \alpha_{2}$ be the frequencies of 1,2 in $s$. It is well known [13] that $\alpha_{2}=\left[0, e_{1}+\right.$ $\left.1, e_{2}, e_{3}, \ldots\right]$.

Consider in particular an immediately periodic continued fraction, $\left[\overline{e_{1}, e_{2}, \ldots, e_{d}}\right]$ (thus $e_{1}>0$, now) and without loss of generality suppose $d$ is even. Then $s$ is directed by $\Delta=w^{\omega}$ where $w=1^{e_{1}} 2^{e_{2}} \cdots 2^{e_{d}}$, i.e., $s$ is the fixed point of $\mu_{w}$. Also consider $\tilde{w}=2^{e_{d}} 1^{e_{d-1}} \cdots 1^{e_{1}}$ and $s^{\prime}$ directed by $\tilde{w}^{\omega}$, i.e., the fixed point of $\mu_{\tilde{w}}$.

The above-mentioned Galois Theorem is as follows.

Theorem 3.1. With $\theta=\left[\overline{e_{1}, e_{2}, \ldots, e_{d}}\right]$ and $\theta^{\prime}=\left[\overline{e_{d}, e_{d-1}, \ldots, e_{1}}\right]$, the algebraic conjugate of the (quadratic by Lagrange's Theorem) number $\theta$ is $-1 / \theta^{\prime}$.

Let us verify that. Set $\theta^{*}=-1 / \theta^{\prime}$. As said above the frequency of 2 in $s$ is $\alpha_{2}=\left[0, e_{1}+1, \overline{e_{2}, e_{3}, \ldots, e_{d}, e_{1}}\right]$ and the frequency of 1 in $s^{\prime}$ is $\alpha_{1}^{\prime}=\left[0, e_{d}+\right.$ $\left.1, \overline{e_{d-1}, e_{d-2}, \ldots, e_{1}, e_{d}}\right]$.

It follows

$$
\alpha_{2}=\frac{1}{\theta+1} \quad \alpha_{1}^{\prime}=\frac{1}{\theta^{\prime}+1}=\frac{\theta^{*}}{\theta^{*}-1} .
$$


Now the vector $\left(\alpha_{1}, \alpha_{2}\right)^{\mathrm{T}}$ is an eigenvector of $M_{w}$ corresponding to the dominating eigenvalue $\xi$ of $M_{w}$ (i.e., $\xi$ is the greater root of $\left.\xi^{2}-\left(m_{11}+m_{22}\right) \xi+1\right)$. Calculation of this vector gives

$$
\alpha_{1}=\frac{\xi-p_{2}}{p_{1}-p_{2}} \quad \alpha_{2}=\frac{p_{1}-\xi}{p_{1}-p_{2}}
$$

and similarly for frequencies $\alpha_{1}^{\prime}, \alpha_{2}^{\prime}$ of 1,2 in $s^{\prime}$. Then using (8) we get

$$
\theta=\frac{\xi-p_{2}}{p_{1}-\xi} \quad \theta^{*}=\frac{p_{2}^{\prime}-\xi}{p_{1}^{\prime}-\xi}
$$

In order to verify that $\theta$ and $\theta^{*}$ are conjugate it suffices to verify that $\theta \theta^{*}$ and $\theta+\theta^{*}$ are rational and this is easy using $\xi^{2}=\left(m_{11}+m_{22}\right) \xi-1$ and $\operatorname{det}\left(M_{w}\right)=$ $m_{11} m_{22}-m_{12} m_{21}=1$.

Remark 3.1. This proof is by far less direct than that of Galois but raises the question of possible generalization to finite alphabets and multidimensional continued fractions.

Acknowledgements. I am most grateful to Valérie Berthé for fruitful discussions and passing me several related papers.

\section{REFERENCES}

[1] C. Allauzen, Une caractérisation simple des nombres de Sturm. J. Th. Nombres Bordeaux 10 (1998) 237-241.

[2] P. Arnoux and G. Rauzy, Représentation géometrique de suites de complexité $2 n+1$. Bull. Soc. Math. France 119 (1991) 199-215.

[3] J. Berstel, Recent results on extensions of Sturmian words. Internat. J. Algebra Comput. 12 (2002) 371-385.

[4] V. Berthé, Autour du système de numération d'Ostrowski. Bull. Belg. Math. Soc. 8 (2001) 209-239.

[5] E. Cahen, Théorie des Nombres. Tome 2, Librairie Scient. A. Hermann, Paris (1924).

[6] A. Carpi and A. de Luca, Harmonic and Gold Sturmian Words, preprint, Dipart. di Mat. G. Castelnuovo, Università degli Studi di Roma La Sapienza, 22/2003 (2003).

[7] M.G. Castelli, F. Mignosi and A. Restivo, Fine and Wilf's theorem for three periods and a generalization of Sturmian words. Theor. Comput. Sci. 218 (2001) 83-94.

[8] X. Droubay, J. Justin and G. Pirillo, Episturmian words and some constructions of de Luca and Rauzy. Theor. Comput. Sci. 255 (2001) 539-553.

[9] E. Galois, Démonstration d'un théorème sur les fractions continues périodiques. Ann. Math. Pures Appl. de M. Gergonne 19 (1829) 294-301.

[10] J. Justin, On a paper by Castelli, Mignosi, Restivo. Theor. Inform. Appl. 34 (2000) 373-377.

[11] J. Justin and G. Pirillo, Episturmian words and episturmian morphisms. Theor. Comput. Sci. 276 (2002) 281-313.

[12] J. Justin and G. Pirillo, Episturmian words: shifts, morphisms and numeration systems. Intern. J. Foundat. Comput. Sci. 15 (2004) 329-348.

[13] M. Lothaire, Algebraic Combinatorics on Words, edited by M. Lothaire. Cambridge University Press. Encyclopedia of Mathematics 90 (2002).

[14] F. Mignosi and L.Q. Zamboni, On the number of Arnoux-Rauzy words. Acta Arith. 101 (2002) 121-129. 
[15] M. Morse and G.A. Hedlund, Symbolic dynamics II: Sturmian trajectories. Amer. J. Math. 62 (1940) 1-42.

[16] G. Rauzy, Nombres algébriques et substitutions. Bull. Soc. Math. France 110 (1982) $147-178$.

[17] G. Rauzy, Mots infinis en arithmétique, in Automata on infinite words, edited by M. Nivat and D. Perrin. Lect. Notes Comput. Sci. 192 (1985) 165-171.

[18] R.N. Risley and L.Q. Zamboni, A generalization of Sturmian sequences, combinatorial structure and transcendence. Acta Arithmetica 95 (2000) 167-184.

[19] N.N. Wozny and L.Q. Zamboni, Frequencies of factors in Arnoux-Rauzy sequences. Acta Arithmetica 96 (2001) 261-278.

[20] L.Q. Zamboni, Une généralisation du théorème de Lagrange sur le développement en fraction continue. C. R. Acad. Sci. Paris I 327 (1998) 527-530. 